\title{
An Effort and Time Based Measure of Usability
}

\author{
Dan Tamir \\ Texas State University \\ San Marcos, TX 78666 \\ $01-512-245-7528$ \\ dt19@txstate.edu
}

\author{
Oleg Komogortsev \\ Texas State University \\ San Marcos, TX 78666 \\ 01-512-245-0349 \\ ok11@txstate.edu
}

\author{
Carl J. Mueller \\ Texas State University \\ San Marcos, TX 78666 \\ 01-512-245-8344 \\ carl.mueller@txstate.edu
}

\begin{abstract}
The hypothesis of this research is that usability relates to the physical effort that is required in order to use software in the accomplishment of interactive tasks. The implications of this hypothesis are significant since effort is an objective quantity that can be measured efficiently in a relatively non-expensive and non-invasive fashion. Based on this hypothesis, this work proposes metrics for measuring operability, learnability, and understandability.

Usability testing is an essential element of a robust validation process. Nevertheless, evaluating software usability is an expensive and time consuming activity, frustrating both developers and managers. Developers are frustrated because it is a critical part of their work; yet, they do not have solid information on how to address it. Managerial frustration lies in the fact that correcting usability defects is an iterative process, and the amount of preparation required can significantly delay a project. Hence, wider utilization of usability testing requires reducing the cost and simplifying the approach.

This paper proposes a framework for measuring effort and using the effort measurements to assess usability. The framework has two main components: One, bases effort on keystrokes and mouse usage; the second uses a model of the eye muscles along with eye-movement tracking to estimate eye effort. The two components are independent, but they could be used in tandem. Tracking time and effort may not be as precise an evaluation of usability. Nevertheless, it can provide a good screening tool to reduce development cost.
\end{abstract}

\section{CATEGORIES AND SUBJECT DESCRIPTORS}

D.2.4 [Software/Program Verification]: Software/Program Verification - Validation

\section{GENERAL TERMS}

Measurement, Human Factors, Verification, Testing

\section{KEYWORDS}

Quality, Metrics, Validation, Usability, Operability, Learnability, Understandability

\section{INTRODUCTION}

Anyone who has ever used a computer can provide a "top ten list" of web sites and programs that are not user-friendly. Poor software usability not only causes user dissatisfaction but also can lead to substantial development cost overruns [10]. Software developers can use a wide variety of tools (prototyping, inspection, usability testing, iterative processes, etc. [11]) to assure the software they produce has good usability. The fact that so many systems present poor usability may point to the conclusion that these techniques do not address the problem efficiently. Furthermore, the challenges presented by usability issues may not lie solely in the tools and techniques used in the development process. Software usability is perceived as a highly subjective attribute of software quality since physiological and psychological characteristics and sociological conditioning heavily influences it. This quality attribute is heavily affected by factors that many software engineers are not familiar with can make them uncomfortable with the entire topic. Our work proposes an objective methodology that can lead to efficient and relatively nonexpensive assessment of usability.

Because it includes a subjective quality attribute, an evaluation of software usability requires observing a number of human subjects using the system. Interpreting these observations necessitates the need of adding a psychologist to the testing team. Some developers do not view usability testing as productive evaluation, because these evaluations usually indicates general area where the subjects had problems and does not necessarily point to a specific solution. This can make developers and managers extremely frustrated, especially when faced with short project deadlines and no way to determine how much time and effort are required to increase the usability. Because of the uncertainty and expense of usability evaluations, some managers are reluctant to include formal usability testing in the development plan. Instead of using testing, these managers prefer to rely on best practices, templates, and inspection to establish software usability. 
The actual challenge of developing usable software may lie in the lack of a clear and concise understanding of what too many software engineers view as a fuzzy concept. Not all authorities on software quality provide a definition of usability. Some authorities recommend usability testing but only provide a check-list of things to investigate $[6,9,2]$; and these authorities are, for the most part, balancing the line between systems with "card input" and interactive systems. Most quality models [4, $10,3,2,8]$ provide a relatively consistent and concise definition of usability, but the attributes used to characterize the many facets of usability are not consistent. This research uses the characterization of usability provided in the ISO/IEC 9126 because it is one of the more recent quality models, it is an industry standard, and it provides a set of measurements and metrics for each quality sub characteristic. Usability in the ISO/IEC 9126 standard is defined as "the capability of the software product to be understood, learned, used, and attractive to the user when used under specified conditions" [4]. The standard also provides the following usability characteristics: Understandability, Learnability; Operability, Attractiveness, and Compliance.

Understandability is the ability of a user to understand the capabilities of the software and if it is suited to accomplish specific goals. It is measured by providing the user with a tutorial or software documentation and then evaluating the users' knowledge to determine the users' level of understanding of the software's functionality, operation, and data input and output [5]. It also recommends using cognitive monitoring techniques to evaluate the user response. Cognitive monitoring techniques are using one-way mirrors or concealed cameras to record the subject's behavior along with evaluation of the findings by a psychology professional.

Learnability describes how easy it is for a user to learn to use the software. For this characteristic, the standard measures how long it takes to the user to learn and perform a task, the number of functions used correctly, and the utility of the help facility [5]. In addition to the measurements, the standard proposes cognitive monitoring techniques.

Operability is the capability of a user to use the software to accomplish a specific goal. To assess operability requires measuring the following characteristics: Operational consistency; Error correction; Error correction in use; Default value availability in use; Message understandability; Selfexplanatory error messages; Operational error recoverability in use; Time between human error operation in use; Undoability; Customizability; Operation procedure reduction; and, Physical accessibility [5]. Some of these metrics are objective measurements, but many require cognitive monitoring techniques to evaluate.

As the name implies, attractiveness is the appeal of the software to a user. Attractiveness is possibly the most subjective of all the usability characteristics, involving not only sociological and psychological issues but gender and personal taste issues. The ISO/IEC 9126 standard characterize attractiveness by providing subjects with a questionnaire to evaluate the interface and by observing subjects customizing the appearance to their satisfaction [5].
Compliance measure how well the software adheres to external and internal rules relating to usability. It is also the most straightforward characteristic to evaluate. Developers compile a list of the required standards, conventions, style guides, and regulations, as well as, through functional testing, verify which standards are meet [5].

Even from this short description of the metrics necessary to evaluate usability, it is apparent that designing a usability test would be an extremely time consuming and thereby expensive task. This is also a test with potentially very high cost which may not identify any specific design or implementation defects. Reducing the high cost of usability testing is difficult because each of the measures proposed by the ISO/IEC standard are good and identify specific problems, and it is not possible to eliminate the use of human test subjects. Another problem with the number of measurements is how to create objective specifications for so many diverse characteristics. Setting objective measurements for all of these metrics would definitely increase the time necessary to specify requirements. What may be possible is to take a slightly different approach to usability testing using techniques that developers and testers are more comfortable with and could administer without requiring cognitive evaluation techniques.

One possible approach to usability testing might be to set a series of goals and to measure the effort and time necessary for a subject to accomplish each goal. If developers estimate the effort and time necessary to complete each goal, then it would be possible to compare the observed effort with the estimated effort. If the observed effort is greater than the estimated effort, then there is a problem requiring further investigation. After identifying the existence of a problem, developers could trace the observation logs to find where the subjects experienced a problem causing the expenditure of additional effort. Trying to evaluate usability from effort and time to complete a goal may be a bit overreaching, but understandability, operability, and learnability should be within the scope of these measurements.

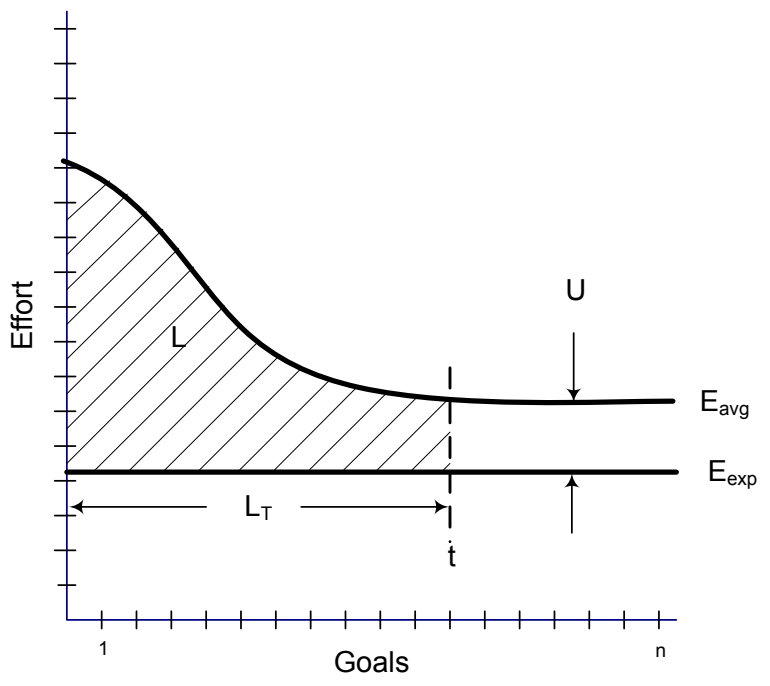

Figure 1 Hypothetical Learning Curve

Using effort to measure operability assumes that software is more operable the less effort it requires to accomplish a specific goal. Consider the following example. Assume a set of $n$ 
subjects selected at random complete a set of $k$ tasks or goals. The objective of each goal is to make travel reservations, and each goal requires about the same effort. After the subjects complete all of the goals, an average of the effort $\left(E_{\text {avg }}\right)$ and the time $\left(T_{a v g}\right)$ for each goal is calculated. The point on a graph, illustrated by Figure 1, where the slope of the average effort $\left(E_{\text {avg }}\right)$ becomes zero ( 0$)$ is the point where the subjects have "learned" how to use the software. The difference between the average effort $\left(E_{\text {avg }}\right)$ and an estimated lower boundary on the effort $\left(E_{\text {exp }}\right)$ can indicate the understandability $(U)$ of the software. The larger the difference, it is believed, the less the user understands about capabilities of the software.

In the simplest form, effort can serve as the basis for static usability measures which are used to tune up the user interface at system design time. In a more advanced form, effort measurement facilities installed at static time remain active and serve as a part of a dynamic and adaptive mechanism for user interface enabling improvement and adaptation to the specific user. This paper concentrates on static usability measurements.

\section{METHODS OF EFFORT EVALUATION}

For this research, effort is the physical activity performed by a person while attempting to accomplish a specific goal. A physical activity could include pressing a key on a keyboard, moving a mouse, pressing a mouse button, or even just the movement of a person's eye. The following sections describe the conversion of physical activities to effort.

\subsection{Effort from Keystroke/Mouse click}

Several informal studies indicate that many system users associate the "physical" effort required for accomplishing tasks with usability of the software. In the case of interactive computer tasks, it may be possible to calculate effort from a weighted sum of mouse clicks, keyboard clicks, mickeys, etc. The term mickey denotes the number of pixels (at the mouse resolution) traversed by the user while moving the mouse from a point $\left(x_{0}, y_{0}\right)$ to a point $\left(x_{1}, y_{1}\right)$. In this research mouse pixels are called mixels.

Our definition of effort uses continuous functions. In practice, given the discrete nature of computer interaction, these measures are quantized by converting integrals to sums. Assume that an interactive task $R$ starts at time $t_{0}$. We define the effort at time $t$ as:

$$
\begin{gathered}
E(t)=\frac{1}{t-t_{0}} \int_{t_{0}}^{t}\left(w_{1} \times m c(t)+w_{2} \times m k(t)+w_{3} \times m i c(t)\right. \\
\left.+w_{4} \times p(t)\right) d t
\end{gathered}
$$

Where: $m c(t), m k(t), m c(t)$ are (respectively) the number of mouse clicks, the number of keyboard clicks, and the number of mixels traversed by the user during the time interval $t-t_{0}$. Furthermore, $p(t)$ is a penalty factor that measures the number of times the user switched from mouse to keyboard or vice versa during the interval. Note that $E(t)$ is a monotonically increasing function.

\subsection{Eye tracking in effort measurement}

Eye tracking is a noninvasive technology, employing Web camera-like devices without any parts affixed to the user's body. An eye tracker can provide usability/effort data even in the absence of overt behavior.
There are two eye tracker-based effort metrics proposed in this paper. The first metric relates the path that the eye travels to the length of the task:

$$
E_{\text {eye_path }}(t)=\frac{\sum_{i=t_{0}}^{t} \sqrt{\left(x_{i+1}-x_{i}\right)^{2}+\left(y_{i+1}+y_{i}\right)^{2}}}{t_{c}-t_{0}}
$$

Where $x_{i}$ and $y_{i}$ are screen eye coordinates (measured in pixels) at a time $i$. The second metric assumes that the force exerted by each extraocular eye muscle is proportional to the eye effort. Hence, the eye effort (normalized to the length of the task) is given by:

$$
E_{\text {eye_muscle }}^{j}(t)=\frac{\sum_{i=t_{0}}^{t_{c}} T_{j}}{t_{c}-t_{0}}
$$

where $T_{j}$ is the extraocular muscle force for the muscle $j$ measured at the moment $i$ [7].

An eye tracker can be employed to provide the values for the force $T j$ based on the model developed by Komogortsev and Khan [7]. This model relates measured eye gaze position and the type of the eye movement to the individual extraocular muscle force [7]. It is important to state that muscular load metric calculated by extraocular muscle force output is more stable and accurate than the metric based on the activity of the limb muscles. This fact comes from the eye anatomy that ensures that the same muscular effort is required for the eye to travel from one point to the next, no matter how many times such movement is repeated as long as the path remains the same. In case the of the hand movement various groups of muscles can be activated with different innervations (the neural stimulation of a muscle) levels for the hand to travel the same point to point path [1]. We have adapted the second model since it provides more information and better precision.

Using the definition of effort related to keyboard / mouse and the definition of eye effort we can provide a measure of effort that combine both. We define the user effort as:

$$
\begin{gathered}
E(t)=\frac{1}{t-t_{0}} \int_{t_{0}}^{t}\left(w_{1} \times m c(t)+w_{2} \times m k(t)+w_{3} \times m i c(t)\right. \\
\left.+w_{4} \times p(t)+w_{5} \times e(t)\right) d t
\end{gathered}
$$

The new equation adds the term $w_{5} \times e(t)$ representing a weighted value of the eye effort measured according to the second eye effort model described above. In systems with no eye-tracking instrumentation, we set $w_{5}$ to 0 .

\section{FORMALIZATION OF ATTRIBUTES}

The hypothesis of this research is that usability, and in specific, operability, learnability, and understandability can be derived from the effort as defined above. One of the goals of this research is to establish the relations between $E(t)$ and traditional measures of operability, learnability and understandability. Additional objectives are to identify the appropriate values for $w_{1}, w_{2}, w_{3}, w_{4}$ and $w_{5}$.

In the current research, we are considering only static measures of usability. This means that the usability of a system is determined at design time and cannot be changed. Another direction of future research is to consider a dynamic scenario where the system adapts to the user and enables user specific improvements in usability at run time. 
Under the assumption of static usability we can ignore the "shape" of the curve of $E(t)$, and only use the "final" effort; that is, the accumulated effort at time of completion of tasks. Let $R$ be a given interactive task. In order to derive the relation between $E(t)$ and static usability, we define the effort associated with a task $R$ in the following way:

$$
\begin{aligned}
\widehat{E_{R}}(t)=\frac{1}{t_{c}-t_{0}} \int_{t_{0}}^{t_{c}}\left(w_{1} \times m c(t)+w_{2} \times m k(t)+w_{3}\right. \\
\left.\times \operatorname{mic}(t)+w_{4} \times p(t)+w_{5} \times e(t)\right) d t
\end{aligned}
$$

where $t_{c}$ is the time of task completion for the task $R$. Note that the division by the factor $t_{c}-t_{0}$ eliminates the dependency of $\widehat{E_{R}}$ in the 'time to completion' factor.

In addition, we assume that $m c\left(t_{0}\right)=m k\left(t_{0}\right)=m i c\left(t t_{0}\right)=$ $p\left(t_{0}\right)=e\left(t_{0}\right)=0$ and define the total effort associated with $R$, to be:

$$
\begin{aligned}
& \widehat{E_{R}}=\frac{1}{t_{c}-t_{0}} \times\left(w_{1} \times m c\left(t_{c}\right)+w_{2} \times m k\left(t_{c}\right)+w_{3} \times \operatorname{mic}\left(t_{c}\right)\right. \\
& \left.+w_{4} \times p\left(t_{c}\right)+w_{5} \times e\left(t_{c}\right)\right)
\end{aligned}
$$

We normalize $\widehat{E_{R}}$, and define $E_{R}$ (the normalized effort associated with $R$ ) by setting $t_{c}=0$ and $t_{0}=1$. At this stage of the research, we assume that $w_{1}=w_{2}=w_{3}=w_{4}=w_{5}=$ 1. Finally, we denote $f(1)$ by $f$ to obtain:

$$
E_{R}=m c+m k+m i c+p+e
$$

where $m c, m k, m i c$, and $p$ denote the total number of mouse clicks, keyboard clicks, mickeys, and switches from mouse to keyboard and keyboard to mouse throughout the process of completing the task $R$.

\subsection{Designer expected effort}

In order to associate $E_{R}$ with operability, learnability, and understandability we define the term: 'the designer expected effort'. Let $R(\bar{x})$ be a task with a parameter vector $\bar{x}$ and let $S$ be a sequence of user interactions that can accomplish $R(\bar{x})$ from scratch. For example, $R(a, b)$ can denote the task of reserving a flight from a city $a$ to a city $b$, then $S$ can include interactions related to subtasks such as checking prices for different airlines, at slightly different arrival / departure times, or from different airports within the cities. The designer expected effort for $R(\bar{x})$ is defined to be:

$$
E_{\text {exp }}=\min _{s \in S} E_{R(\bar{x})}
$$

We assume that when the system is released, the system designer knows $E_{\text {exp }}$. In fact, we would like to assume that the system designer is designing the system in a way that provides the best $E_{\text {exp }}$ over several possible designs. Thus, the designer expected effort is a single number that represents the "ideal" (with respect to minimum effort) way to interact with the system in order to accomplish $R(\bar{x})$.

We now define operability, learnability, and understandability in terms of $E_{\text {exp }}$.

\subsection{Operability}

The operability of a system is measured by $E_{\text {exp }}$ of the system. To elaborate, consider two possible designs $\left(D^{(1)}\right.$ and $\left.D^{(2)}\right)$ of an interactive system for flight reservation enabling the task $R(a, b)$. Let $E_{\text {exp }}^{(1)}$ and $E_{\text {exp }}^{(2)}$ denote the designer expected effort for the designs $D^{(1)}$ and $D^{(2)}$ respectively and assume that $E_{\exp }^{(1)}<E_{\text {exp }}^{(2)}$. Then, per our definition of operability, the operability of design $D^{(1)}$ is better than the operability of design $D^{(2)}$.

\subsection{Learnability}

Learnability is measured by the rate of convergence of user effort to $E_{\text {exp }}$ (see Figure 1). To measure learnability, we consider a large set of tasks of the form $R(\bar{x})$. In the flight reservation system, two of these tasks can be $R(a, b)$ and $R(c, d)$ (reserving a flight from $a$ to $b$ and from $c$ to $d$ ). To simplify the discussion, we assume that the cities are similar and hence, that $E_{\text {exp }}$ is independent of the actual cities. We focus our attention on a user that performs the task $R(u, v)$ repeatedly for many cities $u$ and $v$. We expect that in the beginning of the process the effort related to completing a task $R(u, v)$ will be higher than $E_{\text {exp }}$. As the user repeats $R(u, v)$ (with different cities $u$ and $v$ ), and learns the system, we expect that his effort would converge to $E_{\exp }$.

Figure 1 illustrates the process through a hypothetical learning curve. The figure shows the result of measuring effort made by the user while completing a set of very similar tasks (e.g., flight reservation for many pairs of cities). The figure shows a hypothetical exponential learning curve where the user performance improves along with repeating the task (or with time).

Hence, we can measure learnability as the rate of convergence of the actual user effort $E$ to the ideal effort $E_{\text {exp }}$. Alternatively, we can define learnability in terms of the root mean square error where the error is measured as the difference between the user effort and the designer expected effort at a given task. Yet, another (and similar) measure can be the area of the difference between the learning curve and the curve formed by the fixed line at $y=E_{\text {exp }}$. Figure 2 depicts the learnability (and understandability) curve.

Due to understandability deficiencies, it is possible that the user learning curve does not converge to $E_{\text {exp }}$. Hence, the area is measured up to the point where the user learning curve flattens.

\subsection{Understandability}

Lack of understandability may result in non-efficient usage of the system or using the system for a task that is different than any task defined at design time. In this case, the user effort may converge to a value that is higher than $E_{\text {exp }}$. The difference between the minimal effort value, achieved by the user, and $E_{\text {exp }}$ may be a useful measure for understandability. This is depicted in Figure 2. We will check this assumption through the planned experiments.

System Learnability and Understandability

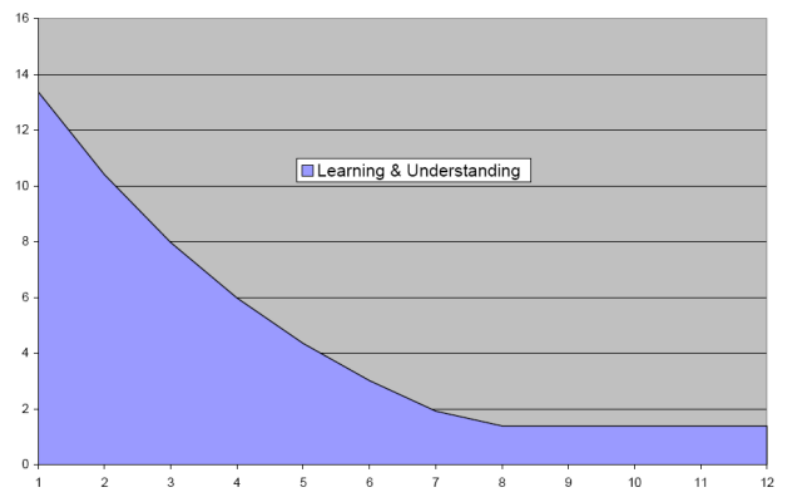


Figure 2 System's Learnability and Understandability

\section{PROPOSED EXPERIMENTS}

$\mathrm{We}$ are developing an infrastructure for instrumenting measurement of user effort related to interactive tasks. At the low level, the infrastructure enables keyboard, mouse, and eye tracking. Raw data collected is interpreted accordingly to reflect effort, operability, learnability, and understandability. In addition, a limited number of informal studies collecting effort data is currently ongoing.

We have selected a travel planning system as the underlying application. The application enables the user to perform four main tasks:

1. One way and round trip flight reservation

2. Hotel reservation

3. Rental car reservation

4. Scheduling of visits to attractions and purchasing tickets if applicable

Each of these tasks requires a multitude of user input parameters such as destination cities, dates, number of travelers, their respective ages, etc. The system interacts with the user, provides a list of "recommendations" (e.g., a list of flights that complies with the user parameters), enables sorting the list, allows changing input parameters, and facilitates selecting list elements. Overall, the system includes numerous options. Generally, specific tasks (for example round trip reservation) can be accomplished through several different interactions sequences, each requiring a different effort.

The system is developed by students as a part of a team project administrated in the "Graphical User Interface" course. The students receive a set of requirements for the reservation capabilities of the system along with requirements for instrumenting the tracking of user activities (mouse clicks, mickey's, etc.). The system requirements include general usability requirements. They do not include specific requirements for "reducing the user effort." Nevertheless, we assume that as a result of the requirement for instrumentation, the students are aware of issues related to user effort and may be taking them into account when they design and implement their system.

The system enables collecting data related to the following events:

- Keyboard click: key clicked, time stamp, relevant widget, and relevant interaction module

- Mouse click: mouse button, time stamp, mixel, relevant widget, and relevant interaction module

- Mouse dragging: A "dense" time sequence of mouse mixels, mapping of mixels to widgets, and to interaction modules performed at the time instances.

- Eye movement: A "dense" time sequence of eye location coordinates, mapping of eye locations to widgets, and to interaction modules performed at the time instances.

The effort has started this semester, and more than one team is working on the project. We expect several implementations of the instrumented reservation system in current and future semesters.
After completing the design and implementation of the travel reservation system, students and instructors operate the developed systems and run a large set of interactive tasks. Raw data related to user activities, described above, is collected, and stored.

The data collected is processed in order to supply higher levels of information including effort, learnability, etc. The data and information gathered are visualized through graphs and tables. We expect to collect information to support and quantify our hypothesis as well as data to be used to calibrate parameters of our models (e.g., the weights associated with the effort function).

\section{CONCLUSIONS AND FUTURE RESEARCH}

We have performed a number of informal studies to assess the hypothesis that usability is a function of effort and time. Clearly, an elaborate set of experiments is required in order to assess the specific nature of the relationships between effort and usability. The research has matured to a level where peer reviews can produce beneficial feedback to make further research more effective.

This paper provides various metrics that describe effort, understandability, learnability and operability. These metrics are defined in terms of basic user input actions such as key strokes, mouse dragging, eye movements, and extraocular muscle force output. Several experiments are proposed for the accurate assessment of metric validity. Successful estimation will allow measuring software operability as a function of physical effort, opening new opportunities for investigation in this domain. We feel that the impact of the proposed research will touch such areas as automated effort estimation, goal design templates, interface quality assessment and others.

There are several directions that the research can take. First, a facility to measure the amount of information that the system provides to the user (i.e., system output) as a function of the user effort can be instrumented. This facility can be used as an additional or alternative operability measure. Another direction for future research includes dynamic and adaptive usable systems. Under this framework, effort-measurement facilities installed at static time remain active and serve as a part of a dynamic and adaptive mechanism for user interface improvement and adaptation to a specific user.

A user pattern of interaction is a sequence of the form $\left\{\left(u_{1}, s_{1}\right),\left(u_{2}, s_{2}\right), \ldots\left(u_{n}, s_{n}\right)\right\}$ where $u_{i}$ is a user activity (e.g., clicking on a push button widget), and $s_{i}$ is a system activity (e.g., producing a list of flights). Under the dynamic model, the system utilizes pattern recognition techniques to identify the repetitive sequences / patterns of interaction. This can be used to set system defaults, reduce required effort, and improve consistency.

Two modes of dynamic systems can be considered. In mode one, referred to as the off-line mode, usability information collected at run time from the current software release is used by the system designers to modify and improve the next software release. In the second mode, referred to as the adaptive mode, the user interface is designed with redundant (extra) widgets. 
These widgets assume the role of dynamically simplifying user interface and reducing user effort. Default values and frequent sequences of interaction are assigned in run time to the extrawidgets in a way that better utilizes default values and converts a sequence of user interaction activities into a single user interaction activity

Using the traditional methods to evaluate usability, there are dozens of indirect measurements requiring analysis. Even with all of these measurements, Tom DeMarco's famous quote: "You can't control what you can't measure", may actually apply to usability with a slight modification "You can't control what you can't directly measure".

\section{REFERENCES}

[1] Bahill, A. T. Development, validation and sensitivity analyses of human eye movement models. CRC Critical Reviews in Bioengineering , 4, 1980.

[2] Boehm, B., et al, Characteristics of Software Quality, North Holland Publishing Co., NY, 1978.

[3] Robert Grady, Practical Software Metrics for Project Management and Process Improvement. Prentice-Hall, 1992.

[4] Anonymous, Software Engineering-Product QualityPart 1: Quality Model. International Standards Organization, Geneva Switzerland, 2001

[5] Anonymous, Software Engineering-Product QualityPart 2: External Metrics. International Standards Organization, Geneva Switzerland, 2001

[6] Kit, E., Software Testing in the Real World, AddisonWesley, Reading, MA. 1995

[7] Komogortsev, O., \& Khan, J. Eye Movement Prediction by Kalman Filter with Integrated Linear Horizontal Oculomotor Plant Mechanical Model. To appear in Eye Tracking Research \& Applications Symposium 2008. at the moment paper accesible at http://www.cs.txstate.edu/ ok11/ETRA08KoKh.pdf.Sa vannah, GA, 2008.

[8] McCall, J.; Richards, P.; Walters, G., "Factors in Software Quality," three volumes, NTIS AD-A049OI4, AD-A049-OI5, AD-A049-055, November 1977.

[9] Myers, G., The Art of Software Testing, John Wiley \& Sons, New York, NY, 1979.

[10] Nielsen, J., Usability Engineering, Academic Press, Inc., San Diego, CA. 1993.

[11] Pressman, R., Software Engineering A Practitioner's Approach, $6^{\text {th }}$ Edition, McGraw-Hill, New York, NY., 2005. 